\title{
Experimental search for color transparency at Jefferson Lab
}

\author{
D. Dutta \\ Duke University, Durham, NC, USA
}

Received: 16 Sep 2003 / Accepted: 14 Nov 2003 /

Published Online: 6 Feb 2004 - (C) Società Italiana di Fisica / Springer-Verlag 2004

\begin{abstract}
Mapping the transition of the strong interaction from the hadronic degrees of freedom to the partonic degrees of freedom of quantum chromodynamics (QCD), is an important goal in intermediate energy nuclear and particle physics. One of the popular approaches used to study the transition between these two regions involves searching for the onset of various phenomena which are naturally predicted in QCD. One such phenomena is Color Transparency (CT), which refers to the suppression of final (and initial) state interactions of hadrons with the nuclear medium in exclusive processes at high momentum transfers. At Jefferson Lab the search for CT has focused on the (e,e'p) reaction. I will review the status of the experimental search for CT covering these experiments and others spanning over a decade. Some of the future experiments being planned for the $12 \mathrm{GeV}$ upgrade of JLab will also be discussed.
\end{abstract}

PACS. 14.20.Dh - 13.40.Gp - 21.10.Ft

\section{Introduction}

Exclusive processes are essential in studies of transitions from non-perturbative to perturbative QCD region. Mapping this transition from the hadronic degrees of freedom to the partonic degrees of freedom is an important goal in intermediate energy nuclear and particle physics. The need for such a mapping arises from the fact that at low energies or long distances the nucleon-meson picture in the standard model of nuclear physics is very successful in describing the overall features of the strong interaction, while at high energies or short distances perturbative QCD (pQCD) with its quark-gluon degrees of freedom allows extremely precise description of the interaction. Unfortunately, there is no clear understanding of how these two regimes are connected. Manifestation of the underlying quark-gluon degrees of freedom of QCD naturally gives rise to a distinct set of phenomena in exclusive processes on nucleons and nuclei. One of the popular methods used to explore the transition region is to look for the onset of such phenomena. One such fundamental predictions of QCD is the phenomena of Color Transparency (CT), it refers to the vanishing of the final (and initial) state interactions of hadrons with the nuclear medium in exclusive processes at high momentum transfer [1. It is based on the idea that, at sufficiently high momentum transfer, the dominant amplitudes for exclusive reactions involve hadrons of reduced transverse size. These small color singlet objects also known as point like configuration (PLC) have long enough lifetimes to be able to pass undisturbed through the nucleus because of reduced interactions with the medium. This is a novel QCD phenomenon which, if observed, would be a clear manifestation of hadrons fluctu- ating to a small size in the nucleus. Moreover, it also contradicts the traditional Glauber multiple scattering theory in the domain of its validity. Nuclear transparency defined as the ratio of the cross section per nucleon for a process on a bound nucleon in the nucleus to the cross section for the process on a free nucleon, is the commonly used observable in searches for this phenomena. Therefore, measurements of nuclear transparency have attracted a significant amount of effort over the last two decades. A clear signature for the onset of $\mathrm{CT}$ would involve a dramatic rise in the nuclear transparency as a function of momentum transfer involved in the process, i.e. a positive slope with respect to the momentum transfer.

\section{The $A\left(e, e^{\prime} p\right)$ reaction}

A number of searches for color transparency have been carried out in the last decade in experiments using the $A(p, 2 p)$ and $A\left(e, e^{\prime} p\right)$ reactions and coherent and incoherent meson production from nuclei [2] - 8]. The $A\left(e, e^{\prime} p\right)$ reaction was first used to measure nuclear transparency at the MIT-Bates lab 9] and the NE-18 experiment at the Stanford Linear Accelerator Center [3] was the first to use this reaction to look for color transparency. These transparency measurements using the $A\left(e, e^{\prime} p\right)$ reaction have been continued at Jefferson Lab (JLab), where the advantage of the continuous wave electron beam has helped improve the precision of the measurements and extend them to higher momentum transfer squared $\left(Q^{2}\right)$. In these reactions an electron beam is incident on a nuclear target and knocks out a proton from within the nucleus. The scattered electron and the knocked out proton are detected 


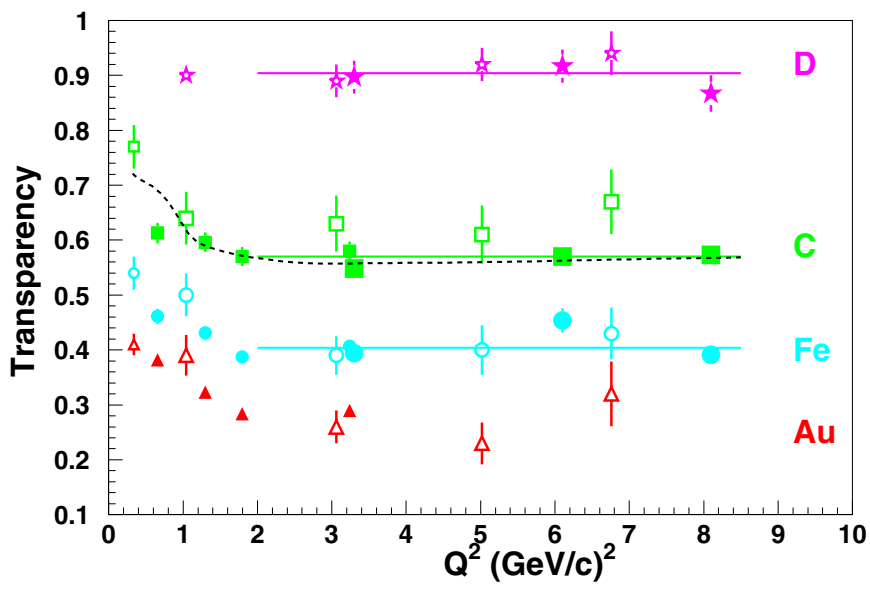

Fig. 1. Nuclear transparency as a function of $Q^{2}$, for ${ }^{2} \mathrm{H}$ (stars), ${ }^{12} \mathrm{C}$ (squares), ${ }^{56} \mathrm{Fe}$ (circles) and ${ }^{197} \mathrm{Au}$ (triangles). The small open symbols are results from MIT-Bates [9], the large open symbols are results from the SLAC experiment NE18 [3], the small solid symbols are results from the earlier JLab experiment 4 and the large solid symbols are results from the later JLab experiment [5]. The dashed line is a Glauber calculation of Pandharipande et al. [10] and the solid lines are fit to a straight line of the results for $Q^{2}>2.0(\mathrm{GeV} / \mathrm{c})^{2}$

in coincidence in a pair of magnetic spectrometers. The proton transparency in the target nucleus is extracted by taking the ratio of the experimental yield to the yield from a Plane Wave Impulse Approximation (PWIA) simulation of the experiment. The PWIA calculation does not include any final state interactions, hence the ratio of the experimental yield to the PWIA yield is a measure of the fraction of the protons which are lost on the way out of the nucleus. The CT prediction implies that for fast protons there will a large decrease in these final state interactions and thus one should observe a dramatic increase in the transparency as a function of momentum transfer squared.

At JLab two $A\left(e, e^{\prime} p\right)$ experiments were performed in experimental Hall $\mathrm{C}$, to measure the nuclear transparency of a wide range of nuclei over a large range of $Q^{2}$. The first of these experiments was performed on ${ }^{1} \mathrm{H},{ }^{12} \mathrm{C},{ }^{56} \mathrm{Fe}$ and ${ }^{197} \mathrm{Au}$ and measured the nuclear transparency up to a $Q^{2}$ of $3.2(\mathrm{GeV} / \mathrm{c})^{2}$. This experiment helped establish a baseline for traditional nuclear physics calculation. The later experiment extended the transparency measurements to a high $Q^{2}$, in search of a deviation from the expectations of the traditional calculations. The second experiment was performed on ${ }^{1} \mathrm{H},{ }^{2} \mathrm{H},{ }^{12} \mathrm{C}$ and ${ }^{56} \mathrm{Fe}$ nuclei and in both experiments the ${ }^{1} \mathrm{H}$ data were used for normalization purposes only. The results of the nuclear transparency measurements with $A\left(e, e^{\prime} p\right)$ experiments from JLab, SLAC and MIT-Bates are shown in Fig. 1] It is clear from Fig. [1 that the transparency is independent of $Q^{2}$ up to a $Q^{2}$ of $8.1(\mathrm{GeV} / \mathrm{c})^{2}$ and the results agree with the expectations of conventional nuclear physics.

One can parametrize the hadron nucleus cross-section as $\sigma_{N}=\sigma_{0} A^{\alpha}$, where $\sigma_{0}$ is the N-N cross-section in free space. Nuclear transparency can thus be parametrized as $T=A^{1-\alpha}$. One can then look at the A dependence of

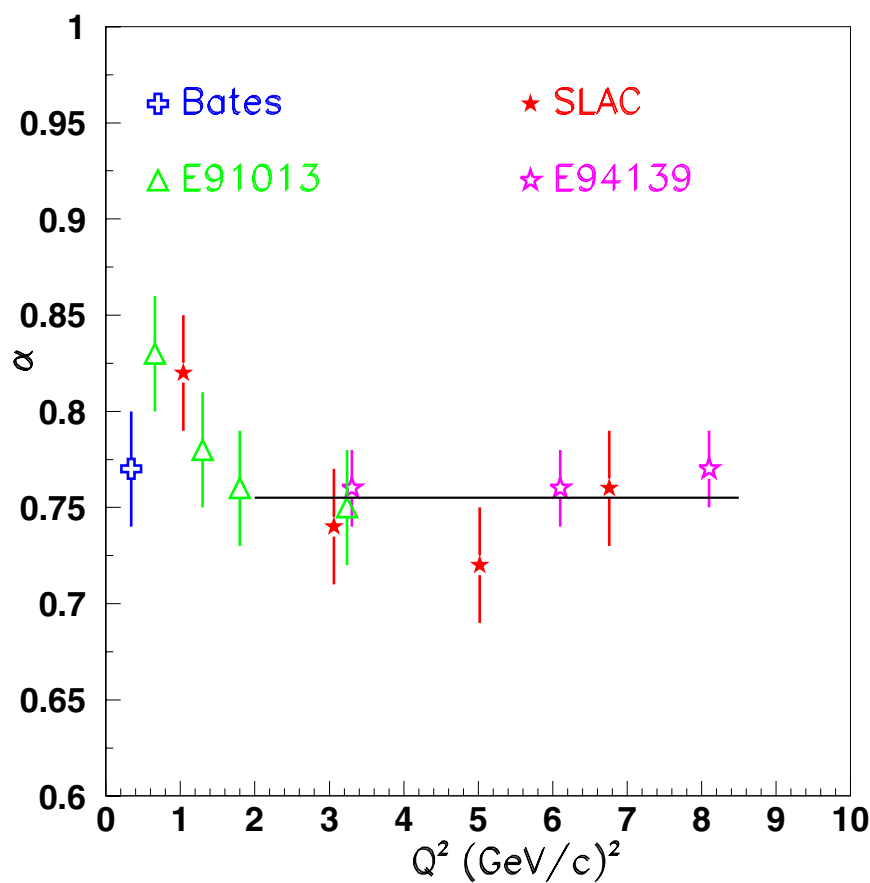

Fig. 2. The parameter $\alpha$ as a function of $Q^{2}$. E91013 and E94139 are the two JLab experiments

the transparency by fitting the transparency at fixed $Q^{2}$ to the form $A^{1-\alpha}$. Figure 2 shows a plot of the parameter $\alpha$ as a function of $Q^{2}$ for all the JLab transparency results. For $Q^{2}>2(\mathrm{GeV} / \mathrm{c})^{2}$ the parameter $\alpha$ is equal to 0.76 which agrees very well with the expectations of conventional nuclear physics model based on just geometrical arguments. Thus these results from JLab conclusively rule out the onset of CT phenomena, up to a $Q^{2}$ of 8.1 $(\mathrm{GeV} / \mathrm{c})^{2}$, for protons produced in exclusive reactions in a nuclear medium.

The $\left(e, e^{\prime} p\right)$ experiments searching for the onset of $\mathrm{CT}$ will be an integral part of the proposed $12 \mathrm{GeV}$ upgrade of JLab and will be able to extend these measurements up to $Q^{2}$ of $18(\mathrm{GeV} / \mathrm{c})^{2}$. The projected results for transparency of ${ }^{12} \mathrm{C}$ from an $\left(e, e^{\prime} p\right)$ experiments with a $11 \mathrm{GeV}$ beam at an upgraded JLab is shown in Fig. 3. It is clear that these future measurements would be able to look for any onset of CT at higher $Q^{2}$ and also be able to resolve the wide range of predictions from various models of the CT effect.

\section{The $\gamma n \rightarrow \pi^{-} p$ reaction}

It is more probable to produce a small transverse size in a $q \bar{q}$ system than in a three quark system, thus one expects an earlier onset of CT for meson production. Support for this comes from recent results on coherent diffractive dissociation of $500 \mathrm{GeV} / \mathrm{c}$ pions into di-jets at Fermilab [8], coherent and incoherent rho mesons production from heavy nuclei in the HERMES experiment 7]. However, none of these results can be considered as conclusive evidence for the onset of CT. 


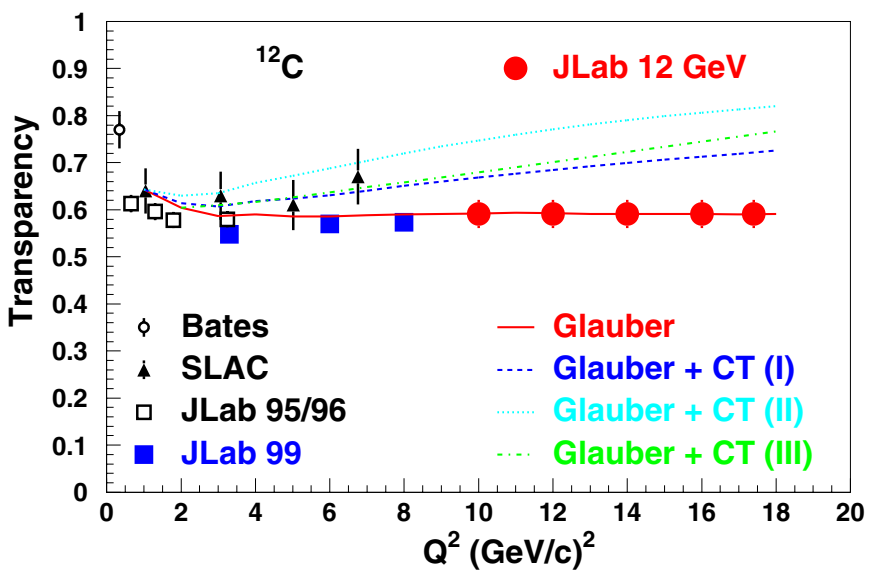

Fig. 3. Projected results from a measurement of transparency of ${ }^{12} \mathrm{C}$ using the $\left(e, e^{\prime} p\right)$ reaction at JLab after the $12 \mathrm{GeV}$ upgrade, shown along with the predictions of various CT calculation, CT(I) [13, CT(II) [11, CT(III) [12] and also a traditional calculation without CT 10

A recent experiment at JLab used the $\gamma n \rightarrow \pi^{-} p$ reaction on ${ }^{4} \mathrm{He}$ to measure the transparency of pions and protons in ${ }^{4} \mathrm{He}$. There are several important advantages to the choice of the ${ }^{4} \mathrm{He}$ nucleus and the $\gamma n \rightarrow \pi^{-} p$ process. Nucleon configurations obtained from the Monte Carlo method based on the exact nuclear ground state wavefunction are available for ${ }^{4} \mathrm{He}$ [14. These configurations along with the elementary hadron-nucleon cross-sections can be used to carry out precise calculations of the nuclear transparency [15] in the framework of Glauber theory [16]. Therefore, precise measurement of nuclear transparency from ${ }^{4} \mathrm{He}$ nuclei is a benchmark test of these traditional nuclear calculations and can be used to explore where the calculations start to break down. Furthermore, light nuclei such as ${ }^{4} \mathrm{He}$ are predicted to be better for the search of CT phenomenon because of their relatively small nuclear sizes, which are smaller than the length scales over which the hadrons of reduced transverse size revert back to their normal sizes. 17, 18.

The experiment was performed in Hall A at JLab. The continuous wave electron beam impinged on a copper radiator to generate an untagged bremsstrahlung photon beam. The combined photon and electron beam was then incident on either helium or liquid deuterium targets. The two High Resolution Spectrometers (HRS) in Hall A, were used to detect the outgoing pions and recoil protons in coincidence. Based on two-body kinematics the incident photon energy is reconstructed for each event using the measured angles and momenta of the $\pi^{-}$and $p$. In case of ${ }^{4} \mathrm{He}$ we also assume that the residual nucleus is ${ }^{3} \mathrm{He}$. The experimental yield is obtained from the reconstructed photon energy spectra by integrating over a $100 \mathrm{MeV}$ window starting $25 \mathrm{MeV}$ below the electron beam energy. This ensures that the contributions from multi-pion processes are negligible. A Monte Carlo simulation of the experiment was used to account for the spectrometer acceptances. As per the definition of nuclear transparency one needs the cross section for $\gamma n \rightarrow \pi^{-} p$ reaction in ${ }^{4} \mathrm{He}$ and in free

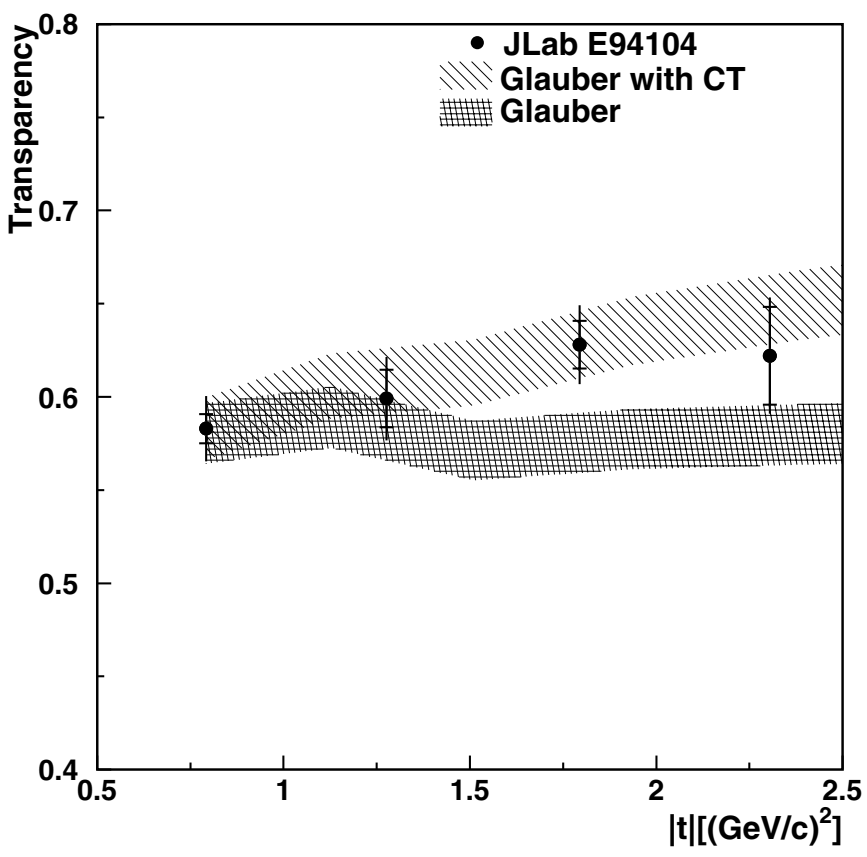

Fig. 4. The nuclear transparency of ${ }^{4} \mathrm{He}\left(\gamma, \mathrm{p} \pi^{-}\right)$at $\theta_{c m}^{\pi}=70^{\circ}$, as a function of momentum transfer square $|t|$. The inner error bars shown are statistical uncertainties only, while the outer error bars are statistical and point-to-point systematic uncertainties $(2.7 \%)$ added in quadrature. In addition there is a $4 \%$ normalization/scale systematic uncertainty which leads to a total systematic uncertainty of $4.8 \%$

space to extract transparency. However, since there are no free neutron targets a deuterium target was used and a correction for deuterium transparency was applied. The transparency was thus extracted from a super-ratio of the data and Monte Carlo yields from ${ }^{4} \mathrm{He}$ and ${ }^{2} \mathrm{H}$ targets corrected for the deuterium transparency.

The extracted nuclear transparency for the ${ }^{4} \mathrm{He}$ target along with calculations is shown in Figs. 4 and 5 . The Glauber calculation use ${ }^{4} \mathrm{He}$ configurations, which are snapshots of the positions of the nucleons in the nucleus, obtained from the variational wave function of Arriaga et al. 14. These contain correlations generated by the Argonne $v_{14}$ and Urbana VIII models of the two-body and three-body nuclear forces respectively. The classical transparency was calculated from these configurations using the method described in [15. The hadron-nucleon total cross-sections were taken from [19. The calculation which includes the CT effect was obtained by repeating the calculation mentioned above with the hadron-nucleon total cross-section modified according to the quantum diffusion model [17. This procedure is also described in [15].

In Figs. 4 and 5 the traditional nuclear physics calculation appears to deviate from the data at the higher energies. The absolute magnitude of the calculations with $\mathrm{CT}$ was normalized to the calculation without $\mathrm{CT}$ at the lowest energy point, however, it is the momentum transfer squared $(|t|)$ dependence of the transparency which is of greater significance. The $|t|$ dependence is not affected by 


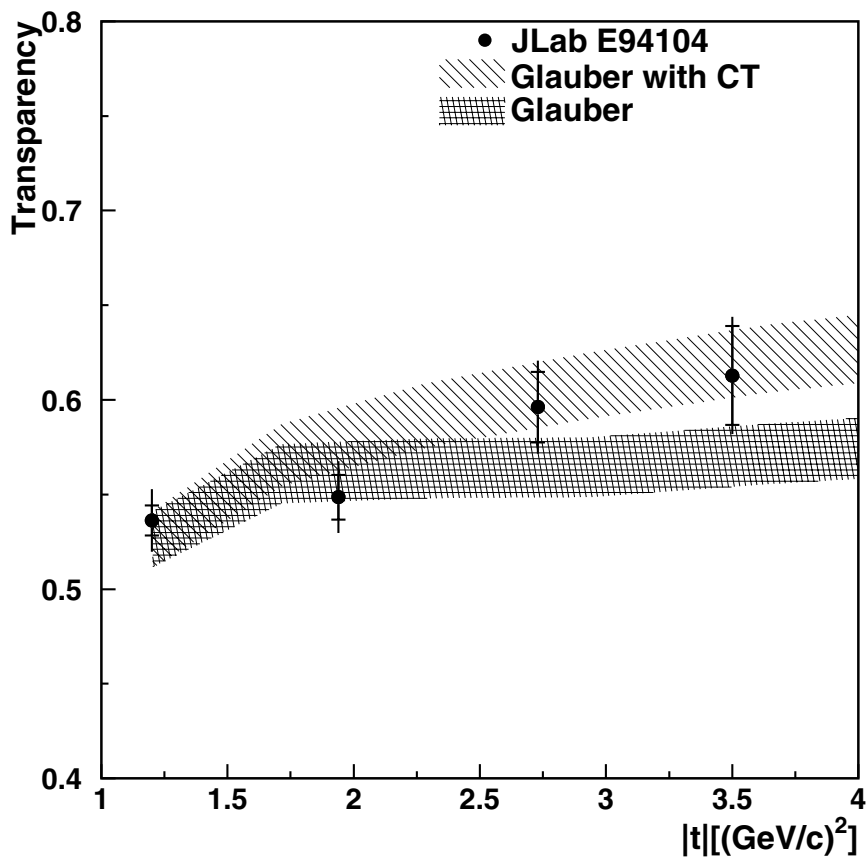

Fig. 5. The nuclear transparency of ${ }^{4} \mathrm{He}\left(\gamma, \mathrm{p} \pi^{-}\right)$at $\theta_{c m}^{\pi}=90^{\circ}$, as a function of momentum transfer square $|t|$. The inner error bars shown are statistical uncertainties only, while the outer error bars are statistical and point-to-point systematic uncertainties $(2.7 \%)$ added in quadrature. In addition there is a $4 \%$ normalization/scale systematic uncertainty which leads to a total systematic uncertainty of $4.8 \%$

the normalization systematic uncertainties. The deviation from Glauber calculation is larger at $\theta_{\mathrm{cm}}^{\pi}=90^{\circ}$, as expected for a CT-like effect, since it is at a higher pion $|t|$. It is also interesting that the results are consistent with the rise expected for $\mathrm{CT}$ at the same photon energy at which the onset of scaling behavior was observed in the cross-section for the $\gamma \mathrm{n} \rightarrow \pi^{-} \mathrm{p}$ and the $\gamma \mathrm{p} \rightarrow \pi^{+} \mathrm{n}$ processes [20]. Thus, these data suggest the onset of deviation from traditional calculations, but future experiments with significantly improved statistical and systematic precision are essential to put these results on a firmer basis.

\section{The $\left(e, e^{\prime} \pi\right)$ reaction}

As mentioned in the previous section it is more probable to produce a small transverse size in a $q \bar{q}$ system than in a three quark system, and thus one expects an earlier onset of CT for meson production. There are some hints of experimental support for these claims, however, none of these results can be considered as conclusive evidence for CT. Thus there is an urgent need for a systematic study of pion transparency over a wide range of $Q^{2}$ and $A$. An experiment to measure pion transparency over a $Q^{2}$ range of $1-5(\mathrm{GeV} / \mathrm{c}){ }^{2}$ on ${ }^{1} \mathrm{H},{ }^{2} \mathrm{H},{ }^{12} \mathrm{C},{ }^{26} \mathrm{Al},{ }^{64} \mathrm{Cu}$ and ${ }^{197} \mathrm{Au}$ has been approved for Hall $\mathrm{C}$ at JLab. This experiment will measure the electroproduction of pion from nuclei by detecting the scattered electron and the knocked out pions in coincidence using the HMS and the SOS spectrome-

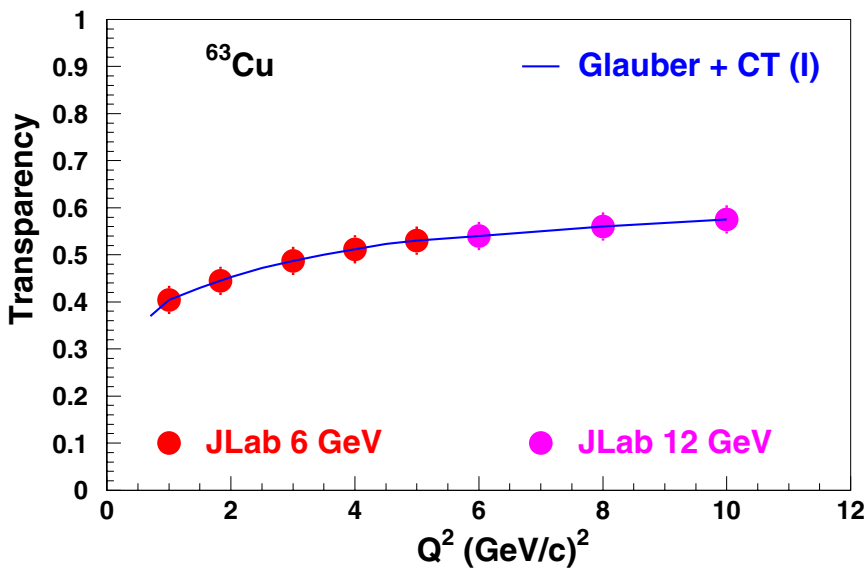

Fig. 6. The projected results for pion transparency in Copper along with the CT calculations of 13. The statistical and systematic uncertainties have ween added in quadrature. Projections for both JLab upgraded to $12 \mathrm{GeV}$ are also shown. Traditional nuclear physics calculations (Glauber calculations) predict an energy independent transparency

ters in Hall C. The pion transparency will be extracted by comparing the pion production from heavy nuclei to that from hydrogen. The $Q^{2}$ interval will be scanned in steps of $1(\mathrm{GeV} / \mathrm{c})^{2}$ to provide a baseline for comparison with traditional nuclear physics calculations at the lower $Q^{2}$ points, while at the higher $Q^{2}$ points it will provide invaluable data on the predicted early onset of CT in $q \bar{q}$ systems. The combined statistical and systematic uncertainty is expected to be $5-7 \%$, while pQCD based calculations predict enhancements as large as $\approx 40 \%$ between $Q^{2}$ of 1 to $5(\mathrm{GeV} / \mathrm{c})^{2}$ for Gold nuclei [13]. The projected results along with a pQCD based calculation [13] is shown in Fig. 6. This experiment would clearly be able to identify an early onset of CT if it exists.

Experiments measuring the pion transparency will greatly benefit form extension to higher $Q^{2}$ range since largest effects are predicted around $Q^{2}$ of $10(\mathrm{GeV} / \mathrm{c})^{2}$ and thus are an integral part of the JLab $12 \mathrm{GeV}$ upgrade program. The projected results for experiments which will be possible at JLab upgraded to $12 \mathrm{GeV}$ are shown in Fig.6.

\section{Conclusions}

Exclusive processes are crucial in studying the transition from the nucleon-meson to the quark-gluon picture. One of the signatures which forms an integral part of such studies is CT. Experiments at JLab has provided some useful clues. They have shown that there is no conclusive evidence for the onset of $\mathrm{CT}$ in $q q q$ systems such as protons, up to $\mathrm{Q}^{2}$ of $8.1(\mathrm{GeV} / \mathrm{c})^{2}$. Another recent experiment 21] has shown interesting deviations form traditional calculations, which support claims for an early onset of CT in $q \bar{q}$ systems. However, future experiments with better statistical and systematic precision in this energy range together with improved theoretical calculations are crucial for confirming these results. The upcoming $\left(e, e^{\prime} \pi\right)$ 
experiment should provide us some valuable information on pion transparency at momentum transfers up to a $\mathrm{Q}^{2}$ value of $5(\mathrm{GeV} / \mathrm{c})^{2}$. The planned upgrade of JLab to 12 $\mathrm{GeV}$ will potentially resolve the issue of the onset of phenomena such as CT by extending the search for $\mathrm{CT}$ to very high $\mathrm{Q}^{2}$.

Acknowledgements. The author is indebted to Drs. R. Ent, H. Gao and R.J. Holt for many fruitful discussions. The author would also like to thank Dr. H. Gao for a careful reading of this manuscript. This work was supported by he U.S. Department of Energy under contract number DE-FG02-03ER41231.

\section{References}

1. S.J. Brodsky and A.H. Mueller: Phys. Lett. B 206, 685 (1988)

2. A.S. Carroll et al.: Phys. Rev. Lett. 61, 1698 (1988); I. Mardor et al.: Phys. Rev. Lett. 81, 5085 (1998); A. Leksanov et al.: Phys. Rev. Lett. 87, 212301-1 (2001)

3. N.C.R. Makins et al.: Phys. Rev. Lett. 72, 1986 (1994); T.G. O'Neill et al.: Phys. Lett. 351, 87 (1995)
4. D. Abbott et al.: Phys. Rev. Lett. 80, 5072 (1998)

5. K. Garrow et al.: Phys. Rev. C 66, 044613 (2002)

6. M.R. Adams et al.: Phys. Rev. Lett. 74, 1525 (1995)

7. A. Airapetian et al.: Phys. Rev. Lett. 90, 052501 (2003)

8. E.M. Aitala et al.: Phys. Rev. Lett. 86, 4773 (2001)

9. G. Garino et al.: Phys. Rev. C 45, 780 (1992)

10. V.R. Pandharipande and S.C. Pieper: Phys. Rev. C 45, 791 (1992); H. Gao, V.R. Pandharipande, and S.C. Pieper: (private communication)

11. L.L. Frankfurt, M.I. Strikman, and M.B. Zhalov: Phys. Rev. C 50, 2189 (1994)

12. N.N. Nikolaev et al.: Phys. Rev. C 50, R1296 (1994)

13. B. Kundu et al.: Phys. Rev. D 62, 113009 (2000)

14. A. Arriaga, V.R. Pandharipande, and R.B. Wiringa: Phys. Rev. C 52, 2362 (1995)

15. H. Gao, R.J. Holt, and V.R. Pandharipande: Phys. Rev. C 54, 2779 (1996)

16. R.J. Glauber: in Lectures in Theoretical Physics, edited by W. E. Brittin et al.: (Interscience, New York, 1959)

17. G.R. Farrar, H. Liu, L.L. Frankfurt, and M.I. Strikman: Phys. Rev. Lett. 61, 686 (1988)

18. Nucl. Phys. A 580, 365 (1994)

19. K. Hagiwara et al.: Phys. Rev. D 66, 010001 (2002)

20. L.Y. Zhu el al.: Phys. Rev. Lett 91, 022003 (2003)

21. D. Dutta et al.: to appear in Phys. Rev. C 